\title{
1 GIGANTEA promotes sorghum flowering by stimulating floral activator gene expression
}

3 Frank G. Harmon ${ }^{1 *}$, Junping $\mathrm{Chen}^{2}$, and Zhanguo Xin ${ }^{2}$

$4 \quad{ }^{1}$ Plant Gene Expression Center, USDA-ARS, Albany, CA 94710 USA and Plant \& Microbial

5 Biology Department, University of California, Berkeley CA 94720 USA

$6 \quad{ }^{2}$ Plant Stress and Germplasm Development Unit, Cropping Systems Research Laboratory, U.S.

7 Department of Agriculture-Agricultural Research Service, Lubbock, Texas 79415 USA

8

9 *Corresponding Author: fharmon@berkeley.edu; (510) 559-5939

10 Junping Chen: Junping.Chen@ars.usda.gov

11 Zhanguo Xin: Zhanguo.Xin@ars.usda.gov 


\section{2 iv. Funding}

13 This work was supported by USDA-ARS CRIS projects 2030-21000-039-00D and 2030-21000-

$14 \quad 049-00 D$ to F.G.H.

16 v. Abstract

17 The C4 grass Sorghum bicolor is an important grain and subsistence crop, animal forage, and 18 cellulosic biofuel feedstock that is tolerant of abiotic stresses and marginal soils. Sorghum is

19 short-day flowering, an obstacle for adaptation as a grain crop but a benefit as a biofuel

20 feedstock. To identify genes underlying sorghum photoperiodic flowering behavior this study

21 characterized the Sbgi-ems 1 nonsense mutation in the sorghum GIGANTEA (SbGI) gene from a

22 sequenced M4 EMS-mutagenized BTx623 population. Sbgi-ems1 plants had reduced stature and

23 leaf blades exhibiting increased lateral growth combined with reduced proximal-distal growth.

24 Mutant plants flowered later than normal siblings under long-day conditions provided by

25 greenhouse or field. Delayed flowering in Sbgi-ems 1 plants accompanied by an increase in

26 internode number, indicating an extended vegetative growth phase prior to flowering. Sbgi-ems 1

27 plants had reduced expression of floral activator genes $S b C O$ and $S b E h d 1$ and downstream FT-

28 like florigen genes $S b F T, S b C N 8$, and $S b C N 12$. Therefore, $S b G I$ accelerates flowering by

29 promotion of $S b C O$ and $S b E h d 1$ expression. Circadian clock-associated genes $S b T O C 1$ and

$30 S b L H Y$ had disrupted expression in Sbgi-ems1 plants. This work demonstrates $S b G I$ is a key

31 upstream activator in the regulatory networks dictating sorghum flowering time and growth, as

32 well as gene expression regulation within the circadian clock. 


\section{5 vi. Key Words}

36 circadian clocks, florigen, flowering time, gene expression, GIGANTEA, Oryza sativa,

37 photoperiodic flowering, Sorghum bicolor, Zea mays

38

39 vii. Acknowledgements

40 Thank you to Emma Kovak and Carine Marshall for feedback and advice. Julianne Elliot and

41 Parkesh Suseendran provided invaluable technical assistance. We thank Lia Poasa and Julie

42 Calfas from the PGEC greenhouse and UC Berkeley Greenhouse Facilities staff Tina Wistrom

43 and Al Hunter for excellent care of plants. This work was supported by USDA-ARS CRIS

44 projects 2030-21000-039-00D and 2030-21000-049-00D to F.G.H.

45

46 Authorship

47 Xin and Chen generated and characterized the sequenced EMS-mutagenized sorghum population

48 containing the Sbgi-ems1 allele. Chen performed early experiments on populations with the Sbgi-

49 ems 1 allele. Harmon conceived, designed, and performed the research. Harmon wrote the

50 manuscript with input from the co-authors.

51

52 Conflicts of Interest: The authors declare no conflicts of interest 


\section{Summary Statement}

54 Sorghum GIGANTEA contributes to flowering time, growth, and the circadian clock with

55 activities opposite to its maize homolog. GI occupies a conserved position within regulatory

56 networks but has plastic activity.

\section{Introduction}

59 Sorghum is a C4 grass native to Africa that is a key grain and subsistence crop, an animal forage, 60 and a promising cellulosic biofuel feedstock. Advantages of sorghum are its high productivity in

61 marginal soils and under arid conditions. Day length is an important signal for triggering

62 flowering in sorghum and as a short-day (SD) plant flowering is promoted when day length falls

63 below a critical threshold (Craufurd et al., 1999; Quinby, 1974). This sensitivity to day length is

64 at once an obstacle for sorghum adaptation as a grain crop and a benefit for its development as a

65 biofuel feedstock (Mullet et al., 2014). To develop sorghums for either purpose, it is important to

66 understand the genes and regulatory pathways that control photoperiodic flowering.

67 Photoperiodic flowering is the regulation of flowering time according to day length. This

68 behavior is enforced by an integrated set of transcriptional and post-transcriptional signaling

69 networks that not only promote flowering under inductive photoperiods but also repress

70 flowering under noninductive photoperiods. A highly conserved point of integration for

71 photoperiodic flowering signals is the CONSTANS (CO) - FLOWERING LOCUS T (FT)

72 regulatory module (Young Hun Song, Shim, Kinmonth-Schultz, \& Imaizumi, 2015), named for

73 genes first discovered in Arabidopsis thaliana. $\mathrm{CO}$ encodes a member of a family of B-box CCT

74 domain transcription factors widely conserved in plants (Griffiths, Dunford, Coupland, \& Laurie,

75 2003; Putterill, Robson, Lee, Simon, \& Coupland, 1995). Arabidopsis FT encodes a member of 
76 the larger plant PEBP-related family conserved throughout flowering plants that contains smaller

77 group of FT-like florigen-related proteins (Danilevskaya, Meng, Hou, Ananiev, \& Simmons,

78 2008; Turck, Fornara, \& Coupland, 2008). According to the florigen hypothesis, a mobile signal

79 originating in leaves transmits the flowering signal to the shoot apical meristem to promote

80 flowering (Pennazio, 2004). Leaf expressed FT-like proteins in Arabidopsis, rice, tomato, and

81 cucurbits serve as molecular florigen signals to trigger the shift from vegetative to floral

82 development at the shoot apical meristem (Jaeger \& Wigge, 2007; Lifschitz et al., 2006; Lin et

83 al., 2007; Notaguchi et al., 2008; Tamaki, Matsuo, Wong, Yokoi, \& Shimamoto, 2007).

84 The primary role of $C O$ is regulation of FT expression and whether $\mathrm{CO}$ protein activates or

85 represses its $F T$ target genes varies among plants. In Arabidopsis, $C O$ activates $F T$ expression to

86 promote flowering under floral inductive long-day (LD) photoperiods (Samach et al., 2000; Y.

87 H. Song, Smith, To, Millar, \& Imaizumi, 2012; Valverde et al., 2004). In contrast, the rice $C O$

88 ortholog Heading date $1(H d l)$ upregulates expression of the FT ortholog Heading date $3 a$

$89(H d 3 a)$ in floral inductive SD photoperiods and represses it in LD (Kojima et al., 2002; Yano et

90 al., 2000). Hdl also represses expression of rice Early heading date 1 (OsEhd1). OsEhd1

91 encodes a B-type response regulator that promotes $H d 3 a$ expression under SD separate from $H d 1$

92 (Doi et al., 2004; Itoh, Nonoue, Yano, \& Izawa, 2010; Zhao et al., 2015). An upstream activator

93 of OsEhd1 expression is Early heading date 2 (Ehd2) encoding a zinc finger transcription factor,

94 which is an ortholog of the maize floral activator INDETERMINATE 1 (ID) (Matsubara et al.,

95 2008). The maize $i d 1$ mutant is very late flowering (Colasanti, Yuan, \& Sundaresan, 1998). The

96 maize florigen-related gene Zea mays CENTRORADIALIS 8 (ZCN8) is a presumed florigen,

97 since silencing ZCN8 expression delays flowering (Meng, Muszynski, \& Danilevskaya, 2011).

98 CONSTANS OF Zea mays1 (CONZ1) is a co-linear ortholog of rice Hdl (Miller, Muslin, \& 
99 Dorweiler, 2008), but genetic and molecular studies have not tested the contribution of CONZ1

100

101

102

103

104

105

106

107

108

109

110

111

112

113

114

115

116

117

118

119

120 to regulation of $Z C N 8$.

Sorghum CONSTANS ( $S b C O)$ acts upstream to promote expression of $S b E h d 1$ and several florigen-related genes in both LD and SD photoperiods (Yang, Weers, Morishige, \& Mullet, 2014). Of the thirteen PEBP-family genes in sorghum, sorghum CENTRORADIALIS 8 (SbCN8) is the co-linear ortholog of maize $Z C N 8$ and $S b F T$ is the co-linear ortholog of rice $H d 3 a(\mathrm{R} \mathrm{L}$ Murphy et al., 2011). An additional PEBP-family gene orthologous between maize and sorghum is $S b C N 12$ (R L Murphy et al., 2011; Yang et al., 2014). Both $S b C N 8$ and $S b C N 12$ possess florigen activity when overexpressed in Arabidopsis (Wolabu et al., 2016). Collectively, SbFT, $S b C N 8, S b C N 12$ are regulated by $S b C O$ and $S b E h d 1$ (Rebecca L. Murphy et al., 2014; Yang et al., 2014), consistent with this set of genes acting as the CO-FT module in sorghum.

An additional repressor of flowering upstream of SbCO is the sorghum PSEUDORESPONSE REGULATOR 37 (SbPRR37) (R L Murphy et al., 2011). SbPRR37 encodes a member of a family of transcriptional repressors originally discovered as core circadian clock genes in Arabidopsis (Farre \& Liu, 2013), but SbPRR37 has no contribution to circadian clock function (R L Murphy et al., 2011). Differentially functional SbPRR37 alleles underlie the flowering time-associated Maturity locus Mal, which has the largest impact on sorghum flowering time (Quinby, 1974). Inactive mal/Sbprr37 alleles confer early flowering in LD conditions and played an important role in early domestication of sorghum (Quinby, 1967). Under LD conditions, SbPRR37 inhibits flowering by repressing expression of flowering activators $S b E h d 1$ and $S b C O$ to ultimately suppress expression of florigen-related genes like $S b F T$, $S b C N 8$, and $S b C N 12$ (R L Murphy et al., 2011). 
GIGANTEA $(G I)$ is a gene identified in early genetic screens for delayed flowering mutants in

122 Arabidopsis (Koornneef, Hanhart, \& van der Veen, 1991; Redei, 1962). GI participates in

123 flowering time control, the circadian clock, and a wide range of other physiological activities

124 (Mishra \& Panigrahi, 2015). In Arabidopsis, GI stimulates flowering by promoting FT

125 expression in LD through direct transcriptional regulation of $F T$ and post-transcriptional

126 inactivation of $C O$ repressors (Park et al., 1999; Sawa \& Kay, 2011; Sawa, Nusinow, Kay, \&

127 Imaizumi, 2007; Suarez-Lopez et al., 2001). Within the circadian clock, GI protein is

128 fundamental to the protein complex that targets the core circadian clock transcriptional repressor

129 TIMING OF CAB 1 (TOC1) for degradation by the ubiquitin-26S proteasome system (Kim et al.,

130 2007; Mas, Kim, Somers, \& Kay, 2003). Tight regulation of TOC1 protein activity is integral to

131 a mutual negative regulatory feedback loop between TOC1 and another core circadian clock

132 gene LATE ELONGATED HYPOCOTYL (LHY) (Alabadi et al., 2001; Gendron et al., 2012;

133 Huang et al., 2012). Sorghum has homologs of both TOC1 and LHY (R L Murphy et al., 2011).

$134 G I$ is also an important component of photoperiodic flowering time networks in grasses. $g i$

135 mutants in rice and maize alter flowering time behavior. Under greenhouse conditions the rice

136 osgi-1 mutant allele delays flowering under SD photoperiods, but not in LD conditions, and only

137 slightly delays flowering in the field (Izawa et al., 2011). OsGI is important for blue light-

138 promoted induction of rice Ehdl expression as part of the mechanism for critical SD day-length

139 recognition (Itoh et al., 2010). Maize has two paralogous GI genes, GIGANTEA1 (GI1) and

140 GIGANTEA2 (Miller 2008; Mendoza 2012). gil mutants flower earlier in LD, but not SD, and

141 have elevated expression of $Z C N 8$ and CONZ1, indicating GI1 is an upstream repressor in LD

142 (Bendix, Mendoza, Stanley, Meeley, \& Harmon, 2013). 
143 The role played by $S b G I$ in regulation of sorghum flowering is not well characterized. A

144 comparative genomic study of 219 African sorghum accessions identified single nucleotide

145 polymorphisms (SNPs) at SbGI significantly associated with photoperiod sensitivity (Bhosale et

146 al., 2012). Two associated SNPs caused non-synonymous amino acid changes and a third

147 represented a frameshift mutation. SbGI expression has a diel rhythm like all known GI genes

148 where peak expression occurs 8 to 10 hours after dawn and this timing is independent of

149 photoperiod (R L Murphy et al., 2011). SbPRR37 does not contribute substantially to regulation

150 of SbGI (R L Murphy et al., 2011).

151 Here we describe a novel mutant allele in the SbGI gene, Sbgi-ems1, identified in a sequenced

152 M4 EMS-mutagenized population (Jiao et al., 2016). Plants carrying this nonsense mutation,

153 which truncates GI protein by two thirds, exhibited a number of alterations in growth and

154 development compared to non-mutant normal siblings. Mutant plants had reduced stature and

155 changes in the orientation of leaf blade growth. The Sbgi-ems 1 allele delayed flowering under

156 LD photoperiod conditions provided by greenhouse or field. Delayed flowering in Sbgi-ems 1

157 accompanied an increase in internode number, indicating an extended vegetative growth phase

158 prior to flowering. This sorghum allele also resulted in reduced expression of the floral activators

$159 S b C O$ and $S b E h d 1$, as well as limited expression of the FT-like florigen genes $S b F T, S b C N 8$, and

$160 S b C N 12$. These observations indicate $S b G I$ promotes $S b C O$ and $S b E h d 1$ expression, which

161 accelerates flowering time under LD photoperiods. Circadian clock gene expression also was

162 disrupted in Sbgi-ems 1 plants. SbTOC1 expression was elevated and $S b L H Y$ expression strongly

163 reduced, consistent with $S b G I$ playing an important role in the regulatory network of the

164 sorghum circadian clock. 
Materials and Methods

Plant stocks and environmental conditions

167 All sorghum lines are the BTx623/ATx623 genetic background. The ARS223 line is from a

168 collection of 256 whole genome sequenced M4 EMS-mutagenized sorghums lines described by

169 Jiao et al. (2016). Plants were screened for the Sbgi-ems 1 mutation in SbGI by Derived Cleaved

170 Amplified Polymorphic Sequences PCR with the primers in Supplemental Table S1. The PCR

171 fragment amplified from the Sbgi-ems 1 locus was resistant to the XcmI restriction enzyme (New

172 England Biolabs) and the product from normal SbGI locus was cleaved by this enzyme.

173 Screening of 24 plants from the M4 ARS223 population yielded one plant heterozygous for the

174 Sbgi-ems 1 allele and this plant was used as pollen donor for a cross to a male sterile ATx623

175 panicle. Progeny of this cross were used for all subsequent experiments.

176 Plants in the greenhouse were grown under LD conditions of 16-hour days and 8-hour nights.

177 Natural sunlight was supplemented with LumiGrow Pro325 LEDs. Daytime temperature was set

178 to $26^{\circ} \mathrm{C}$ and nighttime temperature was set to $20^{\circ} \mathrm{C}$. Seedlings for growth measurements and

179 gene expression were sown in 4-inch peat pots filled with SuperSoil (The Scotts Company),

180 supplemented with a $1 / 2$ teaspoon of 14-14-14 N-P-K slow release fertilizer. Plants for flowering

181 experiments were started in the same fashion then transplanted when seedlings reached the 3-leaf

182 stage (10 days-old) to 13-liter pots filled with corn soil (composed of aged wood fines, green

183 waste compost, fir bark, grape compost, rice hulls, chicken manure, red lava, and sandy loam

184 mixed by American Soil and Stone, Richmond, CA). Greenhouse plants were watered twice

185 daily and received 20-20-20 N-P-K fertilizer once a week after being transplanted to 13-liter

186 pots. Field grown plants were maintained in rows at Oxford tract on the University of California,

187 Berkeley campus and watered to soil saturation once weekly by drip irrigation. For each trial, 
188 field grown plants were started from seed directly at Oxford tract or transplanted as 4-5 leaf

189 individuals ( 2 weeks-old) started is peat pots as above. Plants in the field were grow at the

190 Oxford tract on the UC Berkeley campus from late May 2018 to September 2018.

191

\section{Growth measurements}

193 Plants were grown under greenhouse conditions to the 6-7 leaf stage (4-6 weeks) and measured 194 at this point for height and leaf blade dimensions. Height corresponded to the distance between

195 the soil surface and the collar of the newest fully expanded leaf. The $6^{\text {th }}$ leaf was dissected from 196 the same plants and its length measured from the tip to the ligule. Width was measured at the 197 midpoint of the blade, determined by folding the leaf blade in half. The same measurements were 198 made for the $6^{\text {th }}$ leaf below the flag leaf from post-flowering Sbgi-ems 1 and normal plants.

199 Internodes above the first internode with prop roots were counted on post-flowering plants from 200 flowering time trials.

201

202 Flowering time

203 Plants grown under greenhouse conditions were individually scored for the number of days from 204 sowing to reach boot stage and flowering, while field grown plants were scored for boot stage 205 only, due inhibition of anthesis and stigma exertion by the cool temperatures at the Oxford tract.

206 Boot stage was scored as the first day when the entire flag leaf collar was visible in the leaf

207 whorl. Flowering stage was scored as the first day of anthesis for fertile plants or stigma exertion 208 for male sterile plants. 


\section{Gene Expression}

212 Greenhouse grown $6^{\text {th }}$ leaf stage plants were sampled at 0,8 and 16 hours after dawn. Dawn was

213 when supplemental lights came on at 7 AM. Leaf samples were taken by cutting directly across

214 the $6^{\text {th }}$ leaf ligule with scissors. Two biological replicates were collected for each genotype at

215 each time point. A biological replicate consisted of pooled tissue from three individuals of the

216 same genotype. Leaf samples were flash frozen in liquid nitrogen. After tissue was ground under

217 liquid nitrogen, total RNA was extracted with TRIzol Reagent (ThermoFisher Scientific)

218 according to the manufacturer's recommendations. $1.5 \mu \mathrm{g}$ to total RNA for each sample was

219 treated with dsDNase (ThermoFisher Scientific) to remove contaminating genomic DNA and

220 used as a template for cDNA synthesis with the Maxima H Minus First Strand cDNA synthesis

221 Kit (ThermoFisher Scientific) according to the manufacturer's recommendations. cDNA diluted

222 in half with water served as template for two technical replicate real-time quantitative PCR

223 (qPCR) reactions composed and performed as previously described (Bendix et al., 2013). qPCR

224 reactions for normalization employed PCR primers for 18S RNAs (Supplemental Table S1) and

225 cDNA diluted an additional 1:4000 in water. $\mathrm{C}_{\mathrm{q}}$ values were calculated with the regression

226 function for each primer set in the Bio-Rad CFX Manager Software (BioRad) and relative

227 transcript levels calculated as $2^{\wedge}\left(C_{q}{ }^{18 S_{-}} C_{q}{ }^{\text {experimental }}\right)$. 


\section{Results} gi-ems1 is a nonsense EMS mutation in sorghum $G I$

A single GI gene is present in sorghum genome on the short arm of chromosome 3 (position 3:3,821,973-3,830,666; Sobic.003G040900; SORBI_3003G040900). Publicly available RNAseq analysis shows $S b G I$ is widely expressed in juvenile and adult tissues, with expression higher in leaf, shoot, and root-related tissues compared to flower- and seed-associated tissues

234 (Supplemental Fig. S1A). The sorghum GI protein is over 95\% identical to maize orthologs GI1 235 and GI2 and 68\% identical to the Arabidopsis GI protein (Supplemental Dataset S1).

236 To evaluate the function of $S b G I$, we took advantage of an existing mutant allele in a 237 collection of M4 EMS-mutagenized BTx623 lines described previously (Jiao et al., 2016). The 238 ARS223 line carries an EMS-induced G to A mutation in $S b G I$ at position 5,656 (Fig. 1A). This 239 mutant allele, named here Sbgi-ems1, introduces a premature stop codon in place of a conserved 240 tryptophan (W463*). This allele truncates the normally 1162 residue SbGI protein by two thirds 241 to a 462 amino acid protein (Fig. 1A; Supplemental Dataset S1). Individual plants carrying the

242 Sbgi-ems1 allele were identified in the original ARS223 material by PCR genotyping and the 243 nature of the mutation confirmed by sequencing. One carrier of the Sbgi-ems 1 allele was crossed

244 to a male sterile ATx623 individual to complete backcross 1 (BC1). The BC1F2 and BC1F3

245 generations were used to evaluate the function of SbGI. BC1F3 plants homozygous for Sbgi-

246 ems 1 have reduced overall and peak expression of $S b G I$ compared to normal siblings (Fig. 1B),

247 as is common for nonsense alleles. Rhythmic $S b G I$ expression persists in Sbgi-ems 1 with peak 248 transcript levels occurring 8 hours after dawn similar to normal plants (Fig. 1B). The nature of

249 the Sbgi-ems 1 mutation and the reduction in gene expression indicate this allele causes

250 significant disruption of $S b G I$ activity. 


\section{Visible effects of Sbgi-ems1 on plant growth}

253 Juvenile Sbgi-ems 1 plants exhibited a clear reduction in stature relative to normal siblings. At the

254 6-7 leaf stage, homozygous F3 mutant plants were on average 3-5 cm shorter in two trials under

255 LD greenhouse conditions (Fig. 2A). At the same stage, leaf blade growth was also altered in

256 Sbgi-ems 1 plants. Blades from the $6^{\text {th }}$ leaf from juvenile plants were reduced in length and wider

257 at the midpoint (Fig. 2B; Supplemental Fig. S2A, B), leading to a reduction in the length:width

258 ratio in mutant leaves (Fig. 2C). Mature Sbgi-ems1 individuals at the pre-flowering stage were

259 also visually shorter than normal siblings grown under LD conditions (Fig. 2D). Mature Sbgi-

260 ems 1 plants also exhibited a significant alteration in leaf blade growth, evident as a reduction in

261 the length:width ratio at the midpoint of the $6^{\text {th }}$ leaf below the flag leaf (Fig. 2E). The blade

262 growth change in Sbgi-ems1 mature leaves is mostly due to an increase in blade width

263 (Supplemental Fig. S2 C, D). These observations indicate SbGI activity is important for

264 regulation of both stem and leaf growth in the lateral and proximal-distal directions.

266 Sbgi-ems1 causes delayed flowering

267 The Sbgi-ems1 is associated with delayed flowering time under LD greenhouse and field

268 conditions. To assess whether $S b G I$ contributes to sorghum flowering time, a total of 114

269 individuals from the BC1F2 population were scored for flowering time under LD greenhouse

270 conditions in three separate trials. Flowering time was initially scored as days to anthesis for

271 male fertile plants and days to the exertion of stigma for male sterile plants. The timing of each

272 of these events was indistinguishable within the BC1F2 and BC1F3 groups of plants genotyping

273 as normal at $S b G I$ (Supplemental Fig. S3A). The group of plants homozygous for the Sbgi-ems 1 
274 allele consistently reached anthesis/stigma exertion an average of 35 days later than gil-ems 1

275 heterozygous and normal siblings (Fig. S3A). Heterozygous Sbgi-ems 1 plants reached flowering

276 an average of a week later than normal plants. The late flowering trait tightly co-segregated with

277 the homozygous Sbgi-ems1 genotype in this BC1F2 population (Supplemental Fig. S3B). Two

278 BC1F3 lines each for Sbgi-ems1 and normal plants were selected from this BC1F2 population for

279 further analysis.

280 Delayed flowering time was also evident for Sbgi-ems1 BC1F3 lines. In two separate

281 greenhouse trials, anthesis or stigma exertion for each BC1F3 Sbgi-ems1 population occurred an

282 average of 20 days later than the normal F3 lines under LD conditions (Fig. 3B). The timing of

283 boot stage, which occurs prior to anthesis, was determined in these trials to get a more complete

284 idea of the aspect of flowering changed by Sbgi-ems 1. Similar to the timing of anthesis, boot

285 stage occurred an average of 20 days later in Sbgi-ems 1 plants (Fig. 3B). The timing of boot

286 stage was also determined for the third trial with the BC1F2 population. Sbgi-ems 1 homozygotes

287 in this population were delayed reaching boot stage compared to normal and heterozygous plants

288 (Supplemental Fig. S3C). The average number of days between boot stage and anthesis/stigma

289 exertion for Sbgi-ems 1 and normal plants was not different in all greenhouse trials and in the

290 third trial with the BC1F2 population (Supplemental Fig. S3D). Thus, the flowering time

291 phenotype of Sbgi-ems 1 plants arises from a delay in achieving boot stage, instead of

292 lengthening of the time from boot stage to anthesis.

293 BC1F3 generation Sbgi-ems1 plants produced more internodes than normal siblings. The

294 number of internodes were counted for the F3 plants from the greenhouse flowering trials. Sbgi-

295 ems 1 mutant plants made an average of 2 to 3 internodes than normal F3 plants (Fig. 3C). While

296 Sbgi-ems1 mutants made additional internodes, the length of the main stem of Sbgi-ems1 plants 
remained at or below that attained by normal plants (Fig. 3D). These observations are consistent with an extended vegetative growth phase in Sbgi-ems1 mutant plants, consistent with the Sbgiems 1 allele delaying the timing of the vegetative to floral transition.

Flowering time of BC1F3 Sbgi-ems 1 lines was delayed in field grown plants. To test the determined for F3 Sbgi-ems1 and normal plants grown under LD summer field conditions in reached boot stage more than 25 days later than normal plants (Fig. 3E, F). Clearly, $S b G I$ contributes to the timing of flowering under field conditions, as well as in the greenhouse.

\section{Sbgi-ems1 reduces expression of key flowering time genes}

309 The Sbgi-ems1 allele causes reduced expression of genes that promote flowering. The effect of 310 the Sbgi-ems 1 allele on expression of flowering-related genes was investigated to understand

311 molecular changes underlying delayed flowering. Levels of transcripts for florigen-related genes

$312 S b C N 8, S b C N 12$, and $S b F T$ were assessed at 0, 8, and 16 hours after dawn in month-old normal

313 and Sbgi-ems 1 plants grown under the same greenhouse conditions as the flowering time

314 experiments. In normal plants, $S b C N 8$ and $S b F T$ transcripts reached peak levels 8 hours after

315 dawn (Fig. 4A, B), which coincides with the time of maximal SbGI expression (Fig. 1B).

$316 S b C N 12$ transcripts, on the other hand, were at similar levels in all three time points (Fig. 4C).

317 Sbgi-ems1 plants had reduced levels of $S b C N 8, S b C N 12$, and $S b F T$ transcripts at all three time

318 points. The greatest change for $S b C N 8$ and $S b F T$ occurred 8 hours after dawn, where $S b C N 8$ and 319 SbFT achieved levels 3- and 5-fold lower levels than normal, respectively. SbCN12 levels were 
320 reduced by more than 8 -fold at each time point. These observations are consistent with $S b G I$

321 serving to promote expression of these three florigen-related genes.

322 Since the upstream action of $S b C O$ and Ehd1 control $S b C N 8, S b C N 12$, and $S b F T$, $S b C O$ and

$323 S b E h d 1$ expression was evaluated in normal and Sbgi-ems1 plants. SbCO transcript was present

324 throughout the day in normal plants, with highest levels reached 16 hours after dawn (Fig. 4D).

325 SbEhd1 expression, on the other hand, was biased toward dawn by 2-fold relative to the 16-hour 326 time point (Fig. 4E). In Sbgi-ems 1 plants, $S b C O$ transcript levels were diminished at both 8 and

32716 hours after dawn. In normal plants, SbEhd1 transcript levels were lower in the Sbgi-ems1

328 background at all time points and the greatest reduction of 2-fold occurred at dawn. These results

329 indicate $S b G I$ promotes expression of the two floral activators $S b C O$ and $S b E h d 1$ under LD

330 conditions.

331 The expression of the floral repressor $S b P R R 37$ was also tested to determine whether $S b G I$

332 contributes to its regulation. In normal plants, $S b P R R 37$ transcript levels peaked 16 hours after

333 dawn (Fig. 4F). At all three time points in Sbgi-ems1, the $S b P R R 37$ transcript was below the

334 basal level observed in normal plants at dawn. Thus, $S b G I$ activity contributes to the regulation

335 of SbPRR37.

336 Sbgil-ems 1 did not alter expression of SbID1, a sorghum ortholog of maize ID1, an upstream

337 activator of SbEhd1 that is not directly regulated by SbCO. In normal plants, the highest levels of

338 SbID1 transcript occurred at dawn (time 0 hours) and were reduced by half at the time points 8

339 and 16 hours after dawn (Supplemental Fig. S4A). The Sbgi-ems 1 allele did not change SbID1

340 transcript levels at any time point. Therefore, $S b G I$ is not involved in the regulation of SbIDI. 


\section{3 gi-ems1 disrupts a core circadian clock feedback loop involving $S b L H Y$ and $S b T O C 1$}

344 The Sbgi-ems 1 allele caused disruption of expression for the circadian clock genes $\operatorname{SbLHY}$ and

345 SbTOC1, which are expected to regulate one another in a negative feedback loop. Since GI genes

346 participates in circadian clock function, Sbgi-ems 1 plants were evaluated for changes in

347 expression of the core circadian clock genes SbTOC1 and SbLHY. SbTOC1 transcript was

348 evening-expressed with peak levels occurring 16 hours after dawn in normal greenhouse grown

349 normal plants (Fig. 5B), while $S b L H Y$ transcript was morning-expressed with peak levels

350 occurring at dawn (Fig. 5A). In Sbgi-ems 1 plants, SbTOC1 transcript was elevated relative to

351 normal levels at all time points, particularly at dawn. On the other hand, the $S b L H Y$ transcript

352 was not detectable in mutant plants. These observations indicate $S b G I$ activity is for needed for

353 proper function of an $S b L H Y$ and $S b T O C 1$ regulatory negative feedback loop within the sorghum

354 circadian clock. 


\section{Discussion}

356 Identification of an EMS-derived mutation in the SbGI gene, Sbgi-ems1, allowed us to evaluate

357 the contribution of $S b G I$ to sorghum growth and flowering time. The Sbgi-ems 1 allele is a

358 premature stop codon that truncates GI protein to two thirds of its normal length. Plants

359 homozygous for the Sbgi-ems 1 allele have reduced stature and altered leaf growth. The leaf

360 blade of mutant plants exhibited increased lateral growth and reduced proximal-distal growth,

361 leading to a distortion of the length:width ratio. Sbgi-ems 1 also plants flower later in LD

362 conditions after extended vegetative growth. The delay in flowering is accompanied by a

363 reduction in expression of genes that activate flowering, including the florigen-related genes

$364 S b F T, S b C N 8$ and $S b C N 12$, as well as their upstream regulators $S b C O$ and $S b E h d 1$. Also,

365 expression of circadian clock genes is disrupted by the Sbgi-ems 1 allele. These observations

366 provide insight into the function of $S b G I$, as well as the regulatory networks that determine

367 flowering time in sorghum.

368 The flowering behavior of Sbgi-ems 1 mutant plants indicates $S b G I$ acts early in control of

369 flowering time. Sbgi-ems 1 delayed flowering time under both greenhouse and field conditions

370 when flowering was scored for BC1F2 and BC1F3 individuals as either days to reach boot stage

371 or days to flowering measured as anthesis (for fertile panicles) or stigma exertion (for sterile

372 panicles); however, the time interval between boot stage and anthesis was unchanged in mutant

373 plants relative to normal or heterozygous plants. Thus, the $>20$ additional days Sbgi-ems 1 plants

374 required to reach flowering represents a delay in physiological processes leading up to boot

375 stage. These observations indicate the Sbgi-ems1 allele primarily changes events early in

376 determination of flowering time, not later processes associated with flower development and/or

377 release of pollen/stigma exertion. 
An early for role $S b G I$ in flowering time is consistent with the observation that $S b G I$ is necessary for the proper up-regulation of florigen-related genes $S b F T, S b C N 8$ and $S b C N 12$. In normal plants, $S b F T$ and $S b C N 8$ were rhythmically expressed with peak levels occurring at 8

381 hours after dawn, while $S b C N 12$ expression reached similar levels across the day. The midday

382 peak in $S b F T$ and $S b C N 8$ expression coincided with a similarly timed peak in $S b G I$ expression.

383 Sbgi-ems 1 plants, on the other hand, had reduced $S b C N 12$, SbFT and $S b C N 8$ expression at each

384 time point. Rhythmic expression of SbFT and $S b C N 8$ was notably absent in Sbgi-ems 1, instead 385 each transcript was present at a low constant level at each time. These results are consistent with 386 a requirement for $S b G I$ activity to promote expression of these three florigen-related genes, in 387 particular midday expression $S b F T$ and $S b C N 8$.

$388 S b G I$ appears to promote florigen-related expression through up-regulation of $S b C O$ and $S b E h d 1$ expression. $S b C O$ and $S b E h d 1$ stimulate florigen gene expression under both LD and SD 390 conditions (Rebecca L. Murphy et al., 2014; Yang et al., 2014). Additionally, SbCO activates $391 S b E h d 1$ expression under the all photoperiod conditions. SbCO expression is reduced in the Sbgi392 ems 1 background, indicating $S b G I$ is involved in activation of $S b C O$ at the transcriptional level; 393 however, another possibility that cannot be ruled out is $S b G I$-directed inactivation of an $S b C O$ 394 repressor. Also, SbEhdl expression is reduced in Sbgi-ems1, which could arise from diminished $395 S b C O$ or the absence of direct $S b G I$ up-regulation of $S b E h d 1$. It is notable that the most 396 significant reduction in SbEhd1 expression in Sbgi-ems 1 occurs at dawn. This expression pattern 397 is reminiscent of the loss of morning-induced OsEhd1 expression in the osgi-1 mutant (Itoh et 398 al., 2010). Thus, SbGI may contribute to a regulatory "gate" that promotes $S b E h d 1$ expression in 399 the morning. Sbgi-ems 1 had no impact on expression of SbID1, indicating reduced SbEhd1 400 expression in the mutant background in not due to a lack of up-regulation by SbIDI. 
The flowering time delay in Sbgi-ems1 was unlikely a consequence of reduced SbPRR37 protein-directed repression of $S b E h d 1$ and $S b C O$ even though $S b P R R 37$ expression was diminished in the mutant background. The BTx623/ATx623 genetic background used here

404 carries the Sbprr37-3 allele of mal that encodes inactive PRR37 (R L Murphy et al., 2011).

405 Nevertheless, the change in SbPRR37 expression in Sbgi-ems 1 provides insight into regulation of 406 SbPRR37. Lower SbPRR37 expression in the Sbgi-ems1 background could arise from either loss 407 of direct activation by $S b G I$ or an indirect result of a marked disruption of the circadian clock. In 408 the latter case, strong repression of $S b P R R 37$ may result from elevated SbTOC1 expression. In 409 the Arabidopsis clock system, TOC1 represses $P R R 7$ as part of a timed series of repressive 410 events involving a suite of PRR-family genes (Pokhilko et al., 2012). A reciprocal effect of $411 S b P R R 37$ on SbTOC1 is unlikely, since $\operatorname{SbPRR37}$ is not a component of the sorghum circadian 412 clock as shown by the absence of circadian clock defects in Sbprr37/ma1 alleles (R L Murphy et 413 al., 2011).

414 Comparing the observations here for Sbgi-ems1 to previous work on maize gil mutants 415 highlights interesting differences in the roles played by $G I 1$ and $S b G I$ in these related C4 grasses. 416 The sorghum Sbgi-ems 1 allele and maize gil mutants change growth and flowering time in 417 opposite directions. While the sorghum Sbgi-ems 1 mutant caused significantly later flowering 418 under LD conditions, flowering time is modestly accelerated in maize gil mutants under LD 419 photoperiods (Bendix et al., 2013). Additionally, sorghum Sbgi-ems 1 plants had reduced stature, 420 while maize gil mutants grow taller. It is possible to infer from analysis of gene expression that 421 differences in flowering time between maize and sorghum arise from opposite activities for the 422 cognate $G I$ gene. $S b G I$ serves as an activator of $S b C O$, leading to reduced expression of $S b C O$, $423 S b E H d 1$, and downstream florigen-related genes $S b F T$, $S b C N 8$, and $S b C N 12$ in Sbgi-ems1, while 
424 maize gil is a repressor of CONZ1, leading to upregulation of CONZ1 and ZCN8 in gil mutant

425 backgrounds.

426 In both maize or sorghum, the genesis of growth changes observed in gi mutants remains

427 unclear. Since Arabidopsis GI protein has been implicated in gibberellin (GA) signaling (Tseng

428 et al., 2004) and $O s G I$ is needed for proper regulation of GA biosynthesis genes (Itoh \& Izawa,

429 2011), it is possible that alterations in gibberellin biosynthesis or signaling underlie the growth

430 phenotypes observed in sorghum and maize gi mutants. If this is the case, then the sorghum and

431 maize GI proteins are predicted to have opposite regulatory roles there as well.

432 The Sbgi-ems1 allele disrupted expression of the circadian clock genes $S b L H Y$ and SbTOC1.

433 This is consistent with alteration of a mutual regulatory feedback loop between $S b L H Y$ and

434 SbTOC1 similar to that described in the Arabidopsis circadian clock (Alabadi et al., 2001;

435 Gendron et al., 2012; Huang et al., 2012). Interestingly, TOC1 expression increases and LHY

436 decreases in the osgi-1 mutant background (Izawa et al., 2011). The similar effect of sorghum

437 and rice gi mutants on $S b T O C 1$ and $S b L H Y$ expression indicates comparable circadian clock

438 regulatory networks exist in these grasses. Also, the SbGI and OsGI proteins appear to be

439 involved in transcriptional control of TOC1 expression. By contrast, Arabidopsis GI protein

440 serves to regulate TOC1 protein activity at the post-transcriptional level (Kim et al., 2007;

441 Martin-Tryon, Kreps, \& Harmer, 2007). These observations indicate regulation of TOC1 activity

442 by $G I$ is a conserved feature of plant circadian clocks, but the underlying molecular mechanisms

443 are potentially different between plant species. 


\section{Acknowledgements}

445 Thank you to Emma Kovak and Carine Marshall for feedback and advice. We thank Lia Poasa

446 and Julie Calfas at PGEC greenhouse and Tina Wistrom, and Al Hunter at UC Berkeley

447 Greenhouse Facilities for excellent care of plants. Julianne Elliot and Parkesh Suseendran

448 provided invaluable technical assistance. This work was supported by USDA-ARS CRIS

449 projects 2030-21000-039-00D and 2030-21000-049-00D to F.G.H.

450

\section{Supplemental Files}

452 Supplemental Table S1. Primers used in this study.

453 Supplemental Figure S1. SbGI expression in various sorghum tissues.

454 Supplemental Figure S2. Length and width measurements of leaf blades from juvenile and 455 mature plants.

456 Supplemental Figure S3. Late flowering of Sbgi-ems1 plants arises from delayed boot stage.

457 Supplemental Figure S4. Expression of floral activator SbID1 in Sbgi-ems1.

458 Supplemental Dataset S1. Amino acid alignment of GI proteins from sorghum, maize, and 459 Arabidopsis. 


\section{References}

Alabadi, D., Oyama, T., Yanovsky, M. J., Harmon, F. G., Mas, P., Kay, S. A., ... Kay, S. A. (2001). Reciprocal regulation between TOC1 and LHY/CCA1 within the Arabidopsis circadian clock. Science, 293(5531), 880-883. http://doi.org/10.1126/science.1061320

Bendix, C., Mendoza, J. M., Stanley, D. N., Meeley, R., \& Harmon, F. G. (2013). The circadian clock-associated gene gigantea1 affects maize developmental transitions. Plant, Cell and Environment, 36(7), 1379-1390.

Bhosale, S. U., Stich, B., Rattunde, H. F. W., Weltzien, E., Haussmann, B. I. G., Hash, C. T., ... Parzies, H. K. (2012). Association analysis of photoperiodic flowering time genes in west and central African sorghum [Sorghum bicolor (L.) Moench]. BMC Plant Biology, 12(1), 32. http://doi.org/10.1186/1471-2229-12-32

Colasanti, J., Yuan, Z., \& Sundaresan, V. (1998). The indeterminate gene encodes a zinc finger protein and regulates a leaf-generated signal required for the transition to flowering in maize. Cell, 93(4), 593-603. http://doi.org/10.1016/S0092-8674(00)81188-5

Craufurd, P. Q., Mahalakshmi, V., Bidinger, F. R., Mukuru, S. Z., Chantereau, J., Omanga, P. A., ... Hammer, G. L. (1999). Adaptation of sorghum: Characterisation of genotypic flowering responses to temperature and photoperiod. Theoretical and Applied Genetics, 99(5), 900-911. http://doi.org/10.1007/s001220051311

Danilevskaya, O. N., Meng, X., Hou, Z., Ananiev, E. V., \& Simmons, C. R. (2008). A genomic and expression compendium of the expanded PEBP gene family from maize. Plant Physiology, 146(1), 250-264. http://doi.org/10.1104/pp.107.109538

Doi, K., Izawa, T., Fuse, T., Yamanouchi, U., Kubo, T., Shimatani, Z., ... Yoshimura, A. (2004). Ehd1, a B-type response regulator in rice, confers short-day promotion of flowering and 
controls FT-like gene expression independently of Hd1. Genes \& Development, 18(8), 926936. http://doi.org/10.1101/gad.1189604

Farre, E. M., \& Liu, T. (2013). The PRR family of transcriptional regulators reflects the complexity and evolution of plant circadian clocks. Current Opinion in Plant Biology, 16(5), 621-629. http://doi.org/10.1016/j.pbi.2013.06.015S1369-5266(13)00098-8 [pii]

Gendron, J. M., Pruneda-Paz, J. L., Doherty, C. J., Gross, A. M., Kang, S. E., \& Kay, S. A. (2012). Arabidopsis circadian clock protein, TOC1, is a DNA-binding transcription factor. Proceedings of the National Academy of Sciences of the United States of America, 109(8), 3167-3172. http://doi.org/1200355109 [pii]10.1073/pnas.1200355109

Griffiths, S., Dunford, R. P., Coupland, G., \& Laurie, D. A. (2003). The evolution of CONSTANS-like gene families in barley, rice, and Arabidopsis. Plant Physiology, 131(4), $1855-1867$.

Huang, W., Perez-Garcia, P., Pokhilko, a., Millar, a. J., Antoshechkin, I., Riechmann, J. L., \& Mas, P. (2012). Mapping the core of the Arabidopsis circadian clock defines the network structure of the oscillator. Science, 336(6077), 75-79. http://doi.org/science.1219075 [pii]10.1126/science.1219075

Itoh, H., \& Izawa, T. (2011). A study of phytohormone biosynthetic gene expression using a circadian clock-related mutant in rice. Plant Signaling \& Behavior, 6(12), 1932-6. http://doi.org/10.4161/PSB.6.12.18207

Itoh, H., Nonoue, Y., Yano, M., \& Izawa, T. (2010). A pair of floral regulators sets critical day length for Hd3a florigen expression in rice. Nature Genetics, 42(7), 635-638. http://doi.org/10.1038/ng.606

Izawa, T., Mihara, M., Suzuki, Y., Gupta, M., Itoh, H., Nagano, A. J., ... Nagamura, Y. (2011). 
Os-GIGANTEA confers robust diurnal rhythms on the global transcriptome of rice in the field. Plant Cell, 23(5), 1741-1755. http://doi.org/tpc.111.083238 [pii]

$10.1105 /$ tpc. 111.083238

Jaeger, K. E., \& Wigge, P. A. (2007). FT protein acts as a long-range signal in Arabidopsis. Current Biology, 17(12), 1050-1054.

Jiao, Y., Burke, J., Chopra, R., Burow, G., Chen, J., Wang, B., ... Xin, Z. (2016). A Sorghum Mutant Resource as an Efficient Platform for Gene Discovery in Grasses. The Plant Cell, 28(7), 1551-62. http://doi.org/10.1105/tpc.16.00373

Kim, W.-Y. Y., Fujiwara, S., Suh, S.-S. S., Kim, J., Kim, Y., Han, L., ... Somers, D. E. (2007). ZEITLUPE is a circadian photoreceptor stabilized by GIGANTEA in blue light. Nature, 449(7160), 356-360. http://doi.org/10.1038/nature06132

Kojima, S., Takahashi, Y., Kobayashi, Y., Monna, L., Sasaki, T., Araki, T., \& Yano, M. (2002). $\mathrm{Hd} 3 \mathrm{a}$, a rice ortholog of the ArabidopsisFT Gene, promotes transition to flowering downstream of Hd1 under short-day conditions. Plant and Cell Physiology, 43(10), 10961105. http://doi.org/10.1093/pcp/pcf156

Koornneef, M., Hanhart, C. J., \& van der Veen, J. H. (1991). A genetic and physiological analysis of late flowering mutants in Arabidopsis thaliana. Molecular and General Genetics, 229(1), 57-66. Retrieved from http://www.ncbi.nlm.nih.gov/entrez/query.fcgi?cmd=Retrieve $\& d b=$ PubMed\&dopt=Citation \&list_uids=1896021

Lifschitz, E., Eviatar, T., Rozman, A., Shalit, A., Goldshmidt, A., Amsellem, Z., ... Eshed, Y. (2006). The tomato FT ortholog triggers systemic signals that regulate growth and flowering and substitute for diverse environmental stimuli. Proceedings of the National 
Academy of Scieces, 103(16), 6398-6403. http://doi.org/10.1073/pnas.0601620103

Lin, M.-K., Belanger, H., Lee, Y.-J., Varkonyi-Gasic, E., Taoka, K.-I., Miura, E., ... Lucas, W. J. (2007). FLOWERING LOCUS T Protein May Act as the Long-Distance Florigenic Signal in the Cucurbits. THE PLANT CELL ONLINE, 19(5), 1488-1506. http://doi.org/10.1105/tpc.107.051920

Martin-Tryon, E. L., Kreps, J. A., \& Harmer, S. L. (2007). GIGANTEA acts in blue light signaling and has biochemically separable roles in circadian clock and flowering time regulation. Plant Physiology, 143(1), 473-486. http://doi.org/10.1104/pp.106.088757

Mas, P., Kim, W. Y., Somers, D. E., \& Kay, S. A. (2003). Targeted degradation of TOC1 by ZTL modulates circadian function in Arabidopsis thaliana. Nature, 426(6966), 567-570.

Matsubara, K., Yamanouchi, U., Wang, Z.-X., Minobe, Y., Izawa, T., \& Yano, M. (2008). Ehd2, a Rice Ortholog of the Maize INDETERMINATE1 Gene, Promotes Flowering by UpRegulating Ehd1. Plant Physiology, 148(3), 1425-1435. http://doi.org/10.1104/pp.108.125542

Meng, X., Muszynski, M. G., \& Danilevskaya, O. N. (2011). The FT-likeZCN8 gene functions as a floral activator and is involved in photoperiod sensitivity in maize. Plant Cell, 23(3), 942-960. http://doi.org/tpc.110.081406 [pii]10.1105/tpc.110.081406

Miller, T. A., Muslin, E. H., \& Dorweiler, J. E. (2008). A maize CONSTANS-like gene, conz1, exhibits distinct diurnal expression patterns in varied photoperiods. Planta, 227(6), 13771388. http://doi.org/10.1007/s00425-008-0709-1

Mishra, P., \& Panigrahi, K. C. (2015). GIGANTEA - an emerging story. Frontiers in Plant Science, 6, 8. http://doi.org/10.3389/fpls.2015.00008

Mullet, J., Morishige, D., McCormick, R., Truong, S., Hilley, J., McKinley, B., .. Rooney, W. 
(2014). Energy sorghum--a genetic model for the design of C4 grass bioenergy crops. Journal of Experimental Botany, 65(13), 3479-3489.

http://doi.org/10.1093/jxb/eru229eru229 [pii]

Murphy, R. L., Klein, R. R., Morishige, D. T., Brady, J. A., Rooney, W. L., Miller, F. R., ... Mullet, J. E. (2011). Coincident light and clock regulation of pseudoresponse regulator protein 37 (PRR37) controls photoperiodic flowering in sorghum. Proceedings of the National Academy of Sciences of the United States of America, 108(39), 16469-16474. http://doi.org/1106212108 [pii]10.1073/pnas.1106212108

Murphy, R. L., Morishige, D. T., Brady, J. a., Rooney, W. L., Yang, S., Klein, P. E., \& Mullet, J. E. (2014). Ghd7 (Ma6) Represses Sorghum Flowering in Long Days: Alleles Enhance Biomass Accumulation and Grain Production. The Plant Genome, 7(2), 1-10. http://doi.org/10.3835/plantgenome2013.11.0040

Notaguchi, M., Abe, M., Kimura, T., Daimon, Y., Kobayashi, T., Yamaguchi, A., ... Araki, T. (2008). Long-Distance, Graft-Transmissible Action of Arabidopsis FLOWERING LOCUS T Protein to Promote Flowering. Plant and Cell Physiology, 49(11), 1645-1658. http://doi.org/10.1093/pcp/pcn154

Park, D. H., Somers, D. E., Kim, Y. S., Choy, Y. H., Lim, H. K., Soh, M. S., .. Nam, H. G. (1999). Control of circadian rhythms and photoperiodic flowering by the Arabidopsis GIGANTEA gene. Science, 285(5433), 1579-1582.

Pennazio, S. (2004). "Florigen": An intriguing concept of plant biology. Rivista Di Biologia, 97(1), 33-52. Retrieved from http://www.ncbi.nlm.nih.gov/pubmed/15648209

Pokhilko, A., Fernández, A. P., Edwards, K. D., Southern, M. M., Halliday, K. J., Millar, A. J., ... Millar, A. J. (2012). The clock gene circuit in Arabidopsis includes a repressilator with 
additional feedback loops. Molecular Systems Biology, 8(574), 574.

http://doi.org/10.1038/msb.2012.6

Putterill, J., Robson, F., Lee, K., Simon, R., \& Coupland, G. (1995). The CONSTANS gene of Arabidopsis promotes flowering and encodes a protein showing similarities to zinc finger transcription factors. Cell, 80(6), 847-857. http://doi.org/10.1016/0092-8674(95)90288-0

Quinby, J. R. (1967). The maturity genes of sorghum. In A. G. Norman (Ed.), Advances in Agronomy (Vol. 19, pp. 267-305). New York: Academic Press.

Quinby, J. R. (1974). Sorghum improvement and the genetics of growth. College Station, TX: Texas A\&M University Press. Retrieved from http://books.google.com/books?id=7RchAQAAMAAJ

Redei, G. P. (1962). Supervital mutants of Arabidopsis. Genetics, 47(4), 443-460. Retrieved from http://www.ncbi.nlm.nih.gov/pubmed/17248096

Samach, A., Onouchi, H., Gold, S. E., Ditta, G. S., Schwarz-Sommer, Z., Yanofsky, M. F., \& Coupland, G. (2000). Distinct roles of CONSTANS target genes in reproductive development of Arabidopsis. Science, 288(5471), 1613-1616. http://doi.org/10.1126/science.288.5471.1613

Sawa, M., \& Kay, S. A. (2011). GIGANTEA directly activates Flowering Locus T in Arabidopsis thaliana. Proceedings of the National Academy of Sciences of the United States of America, 108(28), 11698-11703. http://doi.org/1106771108 [pii]10.1073/pnas.1106771108

Sawa, M., Nusinow, D. A., Kay, S. A., \& Imaizumi, T. (2007). FKF1 and GIGANTEA complex formation is required for day-length measurement in Arabidopsis. Science, 318(5848), 261265. http://doi.org/1146994 [pii]10.1126/science.1146994 
Song, Y. H., Shim, J. S., Kinmonth-Schultz, H. A., \& Imaizumi, T. (2015). Photoperiodic

Flowering: Time Measurement Mechanisms in Leaves. Annual Review of Plant Biology, 66(1), 441-464. http://doi.org/10.1146/annurev-arplant-043014-115555

Song, Y. H., Smith, R. W., To, B. J., Millar, A. J., \& Imaizumi, T. (2012). FKF1 Conveys

Timing Information for CONSTANS Stabilization in Photoperiodic Flowering. Science, 336(6084), 1045-1049. http://doi.org/10.1126/science.1219644

Suarez-Lopez, P., Wheatley, K., Robson, F., Onouchi, H., Valverde, F., Coupland, G., ...

Coupland, G. (2001). CONSTANS mediates between the circadian clock and the control of flowering in Arabidopsis. Nature, 410(6832), 1116-1120. http://doi.org/10.1038/35074138

Tamaki, S., Matsuo, S., Wong, H. L., Yokoi, S., \& Shimamoto, K. (2007). Hd3a protein is a mobile flowering signal in rice. Science, 316(5827), 1033-1036.

Tseng, T.-S. S., Salomé, P. A., McClung, C. R., Olszewski, N. E., Salome, P. A., McClung, C. R., \& Olszewski, N. E. (2004). SPINDLY and GIGANTEA interact and act in Arabidopsis thaliana pathways involved in light responses, flowering, and rhythms in cotyledon movements. Plant Cell, 16(6), 1550-1563. http://doi.org/10.1105/tpc.019224

Turck, F., Fornara, F., \& Coupland, G. (2008). Regulation and identity of florigen:

FLOWERING LOCUS T moves center stage. Annual Review of Plant Biology, 59(1), 573594. http://doi.org/10.1146/annurev.arplant.59.032607.092755

Valverde, F., Mouradov, A., Soppe, W., Ravenscroft, D., Samach, A., \& Coupland, G. (2004).

Photoreceptor regulation of CONSTANS protein in photoperiodic flowering. Science, 303(5660), 1003-1006. http://doi.org/10.1126/science.1091761

Wolabu, T. W., Zhang, F., Niu, L., Kalve, S., Bhatnagar-Mathur, P., Muszynski, M. G., \& Tadege, M. (2016). Three FLOWERING LOCUS T-like genes function as potential 
florigens and mediate photoperiod response in sorghum. New Phytologist, 210(3), 946-959. http://doi.org/10.1111/nph.13834

Yang, S., Weers, B. D., Morishige, D. T., \& Mullet, J. E. (2014). CONSTANS is a photoperiod regulated activator of flowering in sorghum. BMC Plant Biology, 14(1), 148.

http://doi.org/10.1186/1471-2229-14-148

Yano, M., Katayose, Y., Ashikari, M., Yamanouchi, U., Monna, L., Fuse, T., ... Sasaki, T. (2000). Hd1, a major photoperiod sensitivity quantitative trait locus in rice, is closely related to the Arabidopsis flowering time gene CONSTANS. Plant Cell, 12(12), 24732484.

Zhao, J., Chen, H., Ren, D., Tang, H., Qiu, R., Feng, J., ... Guo, J. (2015). Genetic interactions between diverged alleles of Early heading date 1 (Ehd1) and Heading date 3a (Hd3a)/ RICE FLOWERING LOCUS T1 (RFT1) control differential heading and contribute to regional adaptation in rice (Oryza sativa). The New Phytologist, 208(3), 936-48.

http://doi.org/10.1111/nph.13503 


\section{Figure Legends}

\section{Figure 1. The Sbgi-ems 1 mutant is a nonsense allele that results in reduced $S b G I$}

expression. A) Diagram of SbGI gene (Sobic.003G040900; SORBI_3003G040900) where boxes indicate exons and lines introns. Red coloring indicates coding sequence with the arrow at start codon and white regions indicating 5'- and 3'-UTRs. Vertical line above indicates the position and nature of the Sbgi-ems1. B) Transcript levels for SbGI in leaves of normal (white bars) and Sbgi-ems1 (black bars) BC1F3 plants at $6^{\text {th }}$ leaf stage grown under LD conditions. Time after dawn is the number of hours after lights on in the morning. Values are the average of two biological replicates normalized to the time point from normal plants with highest transcript levels. Error bars represent the range of two biological replicates.

\section{Figure 2. Sbgi-ems1 reduces plant stature and changes the orientation of leaf growth.}

Height (A), representative $6^{\text {th }}$ leaves $(B)$, and length:width ratio of blades from $6^{\text {th }}$ leaves $(C)$ of BC1F3 juvenile normal (circles) and Sbgi-ems 1 (triangles) plants grown to the 6-7 leaf stage. D) Representative 2-month-old pre-flowering normal and Sbgi-ems1 plants. E) Length:width ratio of blades from $6^{\text {th }}$ leaf below flag leaf on $\mathrm{BC} 1 \mathrm{~F} 3$ mature post-flowering normal (circles) and Sbgi-ems1 (triangles) plants. All plants were grown under LD greenhouse conditions.

Length:width ratio was calculated from length and width measurements in Supplemental Figure S2. All measurements are shown from two separate trials, bar represents the average of measurements. Statistical significance is indicated according to a two-tailed unpaired t-tests with Welch's correction at $\mathrm{P}$ value $<0.0001(* * * *),<0.001(* * *),<0.01(* *)$ and $<0.05(*)$. 
Figure 3. Sbgi-ems 1 mutants are late flowering and produce more internodes prior to

flowering. A) Flowering time for BC1F2 population for normal (circles), heterozygous Sbgiems 1/SbGI (squares), and Sbgi-ems 1 (triangles) plants grown under LD greenhouse conditions determined as days to anthesis (fertile plants) or stigma exertion (male sterile plants). B) Flowering time for BC1F3 normal (circles) and Sbgi-ems1 (triangles) plants grown under LD greenhouse conditions determined as days to boot stage (DTB) and days to anthesis or stigma exertion (DTA/S). C) Number of internodes (Nodes) above prop roots produced by normal (circles) and Sbgi-ems 1 (triangles) BC1F3 plants from flowering time experiments. D) Representative main stems after leaf removal from normal and Sbgi-ems 1 plants from flowering time experiments under greenhouse conditions. E) Flowering time for BC1F3 normal (circles) and Sbgi-ems1 (triangles) plants grown under summer field conditions determined as days to boot stage. F) Pictures of representative 3-month-old normal and Sbgi-ems 1 plants grown in the field. All measurements are shown from two separate trials, bar represents the average of measurements. Statistical significance is indicated according to a two-tailed unpaired t-tests with Welch's correction at $\mathrm{P}$ value $<0.0001(* * * *),<0.001(* * *),<0.01(* *)$ and $<0.05(*)$.

Figure 4. Sbgi-ems1 alters flowering time gene expression patterns and levels. Transcript levels for $S b C N 8$ (A), $S b F T$ (B), $S b C N 12$ (C), $S b C O$ (D), $S b E h d 1$ (E), and $S b P R R 37$ (F) in leaves of normal (white bars) and Sbgi-ems1 (black bars) BC1F3 plants at $6^{\text {th }}$ leaf stage grown under LD conditions. Time after dawn is the number of hours after lights on in the morning. Values are the average of two biological replicates normalized to the time point from normal plants with highest transcript levels. Error bars represent the range of two biological replicates. 
Figure 5. Sbgi-ems1 disrupts $S b L H Y$ and $S b T O C 1$ expression. Transcript levels for $S b L H Y$ (A) and SbTOC1 (B) in leaves of normal (white bars) and Sbgi-ems1 (black bars) BC1F3 plants at $6^{\text {th }}$ leaf stage grown under LD conditions. Time after dawn is the number of hours after supplemental lights came on in the morning. Values are the average of two biological replicates normalized to the time point from normal plants with highest transcript levels. Error bars represent the range of two biological replicates. 


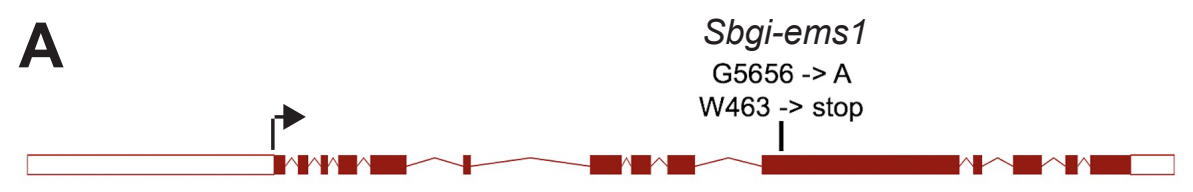

SORBI_3003G040900 | Sobic.003G040900

B

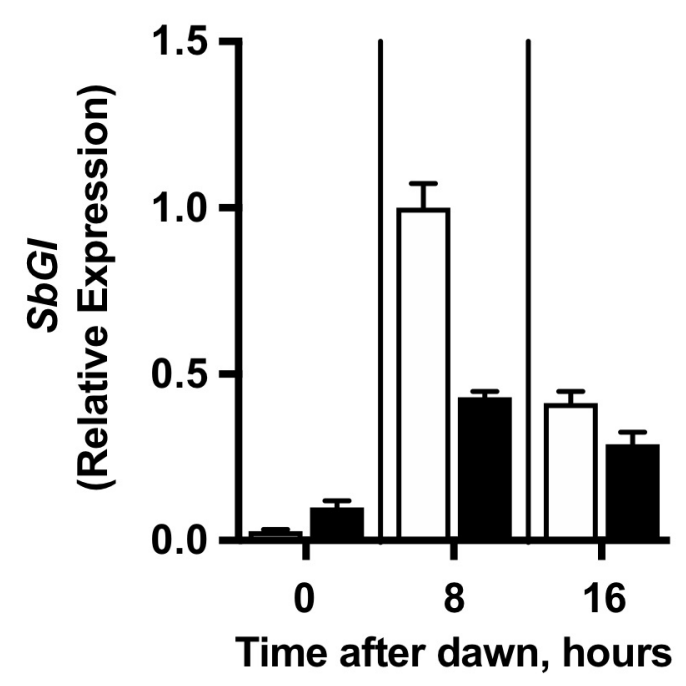

Figure 1. The Sbgi-ems1 mutant is a nonsense allele that results in reduced $S b G I$ expression. A) Diagram of SbGI gene (Sobic.003G040900; SORBI_3003G040900) where boxes indicate exons and lines introns. Red coloring indicates coding sequence with the arrow at start codon and white regions indicating 5'- and 3'-UTRs. Vertical line above indicates the position and nature of the Sbgi-ems1. B) Transcript levels for SbGI in leaves of normal (white bars) and Sbgi-ems1 (black bars) BC1F3 plants at $6^{\text {th }}$ leaf stage grown under LD conditions. Time after dawn is the number of hours after lights on in the morning. Values are the average of two biological replicates normalized to the time point from normal plants with highest transcript levels. Error bars represent the range of two biological replicates. 

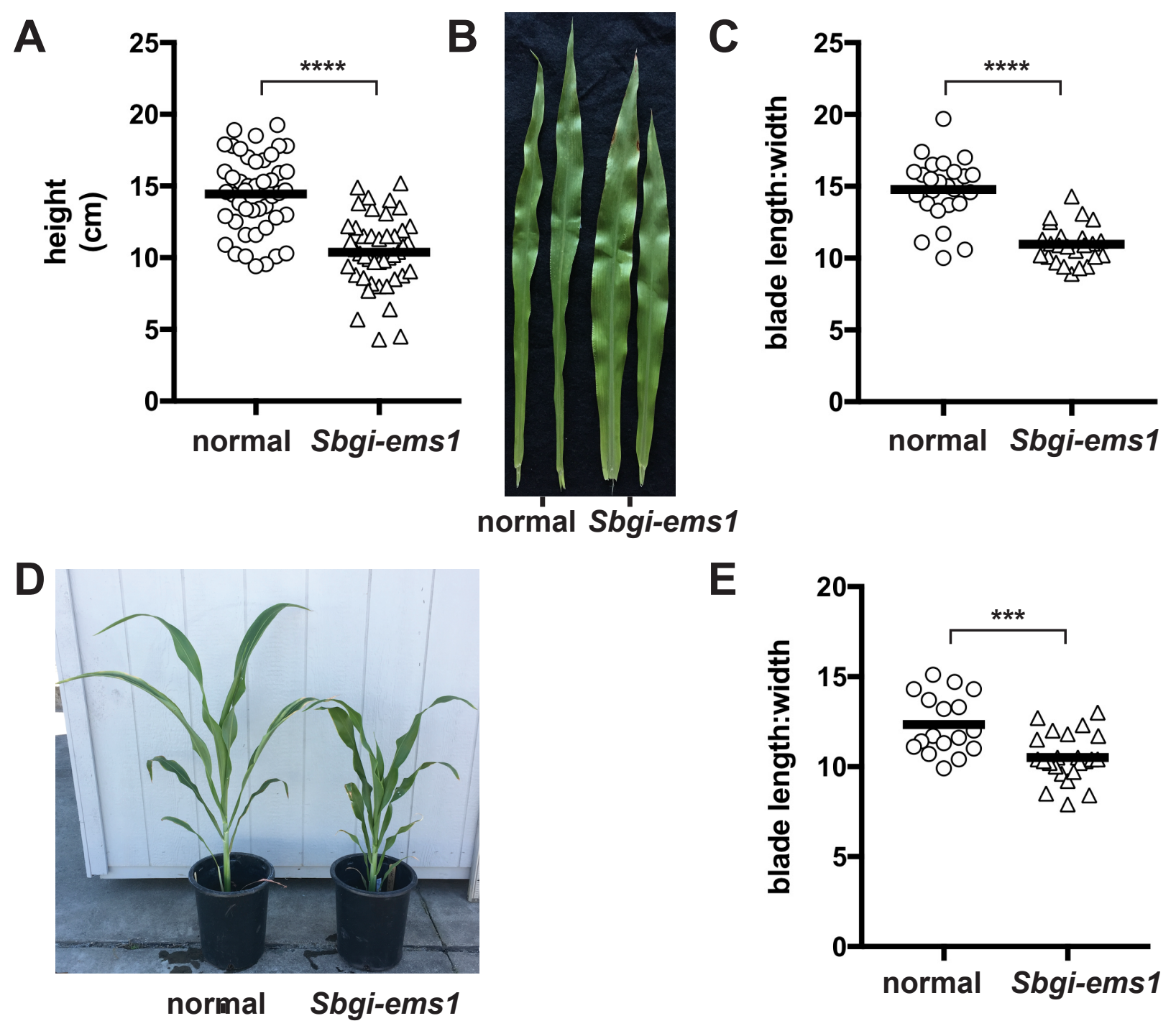

Figure 2. Sbgi-ems1 reduces plant stature and changes the orientation of leaf growth. Height (A), representative $6^{\text {th }}$ leaves $(B)$, and length:width ratio of blades from $6^{\text {th }}$ leaves (C) of BC1F3 juvenile normal (circles) and Sbgi-ems1 (triangles) plants grown to the 6-7 leaf stage. D) Representative 2-month-old pre-flowering normal and Sbgiems1 plants. E) Length:width ratio of blades from $6^{\text {th }}$ leaf below flag leaf on BC1F3 mature post-flowering normal (circles) and Sbgi-ems1 (triangles) plants. All plants were grown under LD greenhouse conditions. Length:width ratio was calculated from length and width measurements in Supplemental Figure 2. All measurements are shown from two separate trials, bar represents the average of measurements. Statistical significance is indicated according to a two-tailed unpaired t-tests with Welch's correction at $\mathrm{P}$ value $<0.0001\left(^{* * *}\right),<0.001\left(^{* * *}\right),<0.01\left(^{* *}\right)$ and $<0.05\left(^{*}\right)$. 

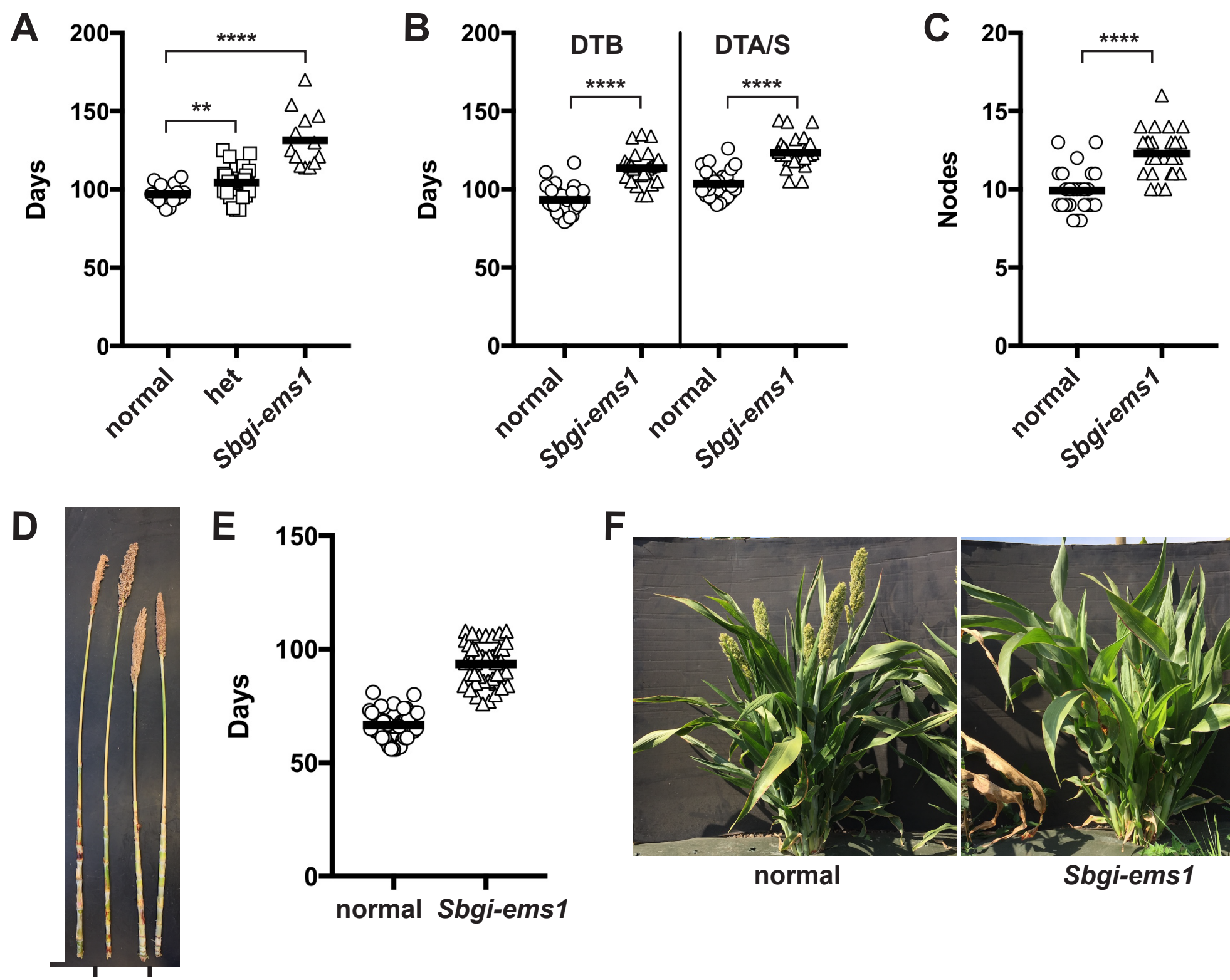

normal

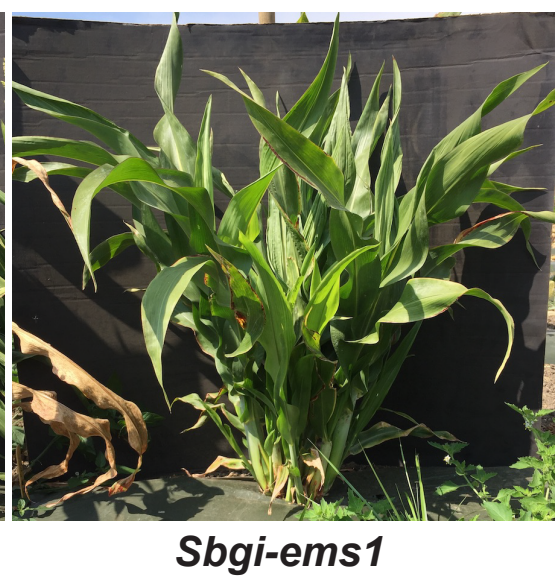

normal Sbgi-ems1

Figure 3. Sbgi-ems1 mutants are late flowering and produce more internodes prior to flowering. A) Flowering time for BC1F2 population for normal (circles), heterozygous Sbgi-ems1/SbGI (squares), and Sbgi-ems1 (triangles) plants grown under LD greenhouse conditions determined as days to anthesis (fertile plants) or stigma exertion (male sterile plants). B) Flowering time for BC1F3 normal (circles) and Sbgiems1 (triangles) plants grown under LD greenhouse conditions determined as days to boot stage (DTB) and days to anthesis or stigma exertion (DTA/S). C) Number of internodes (Nodes) above prop roots produced by normal (circles) and Sbgi-ems 1 (triangles) BC1F3 plants from flowering time experiments. D) Representative main stems after leaf removal from normal and Sbgi-ems1 plants from flowering time experiments under greenhouse conditions. E) Flowering time for BC1F3 normal (circles) and Sbgi-ems1 (triangles) plants grown under summer field conditions determined as days to boot stage. F) Pictures of representative 3-month-old normal and Sbgi-ems1 plants grown in the field. All measurements are shown from two separate trials, bar represents the average of measurements. Statistical significance is indicated according to a two-tailed unpaired t-tests with Welch's correction at $\mathrm{P}$ value $<0.0001{ }^{\left({ }^{* * *}\right)},<0.001$ $\left({ }^{* * *}\right),<0.01\left(^{* *}\right)$ and $<0.05\left(^{*}\right)$. 


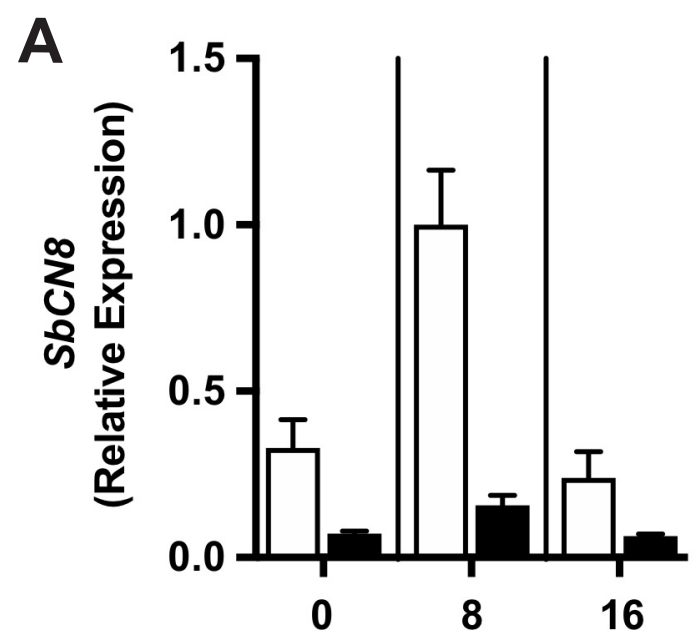

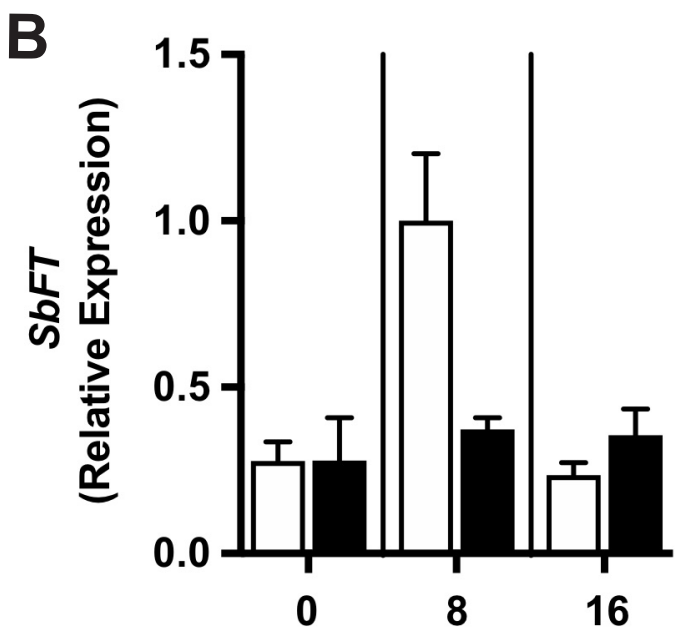

Time after dawn, hours

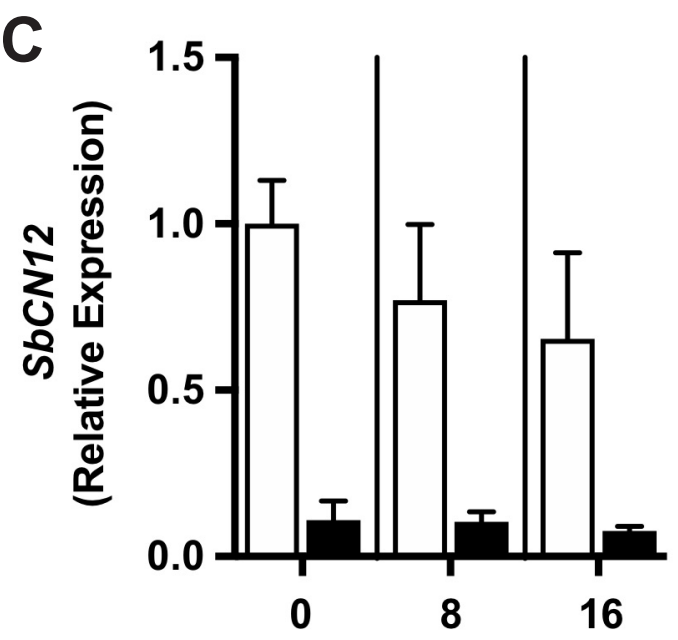

Time after dawn, hours

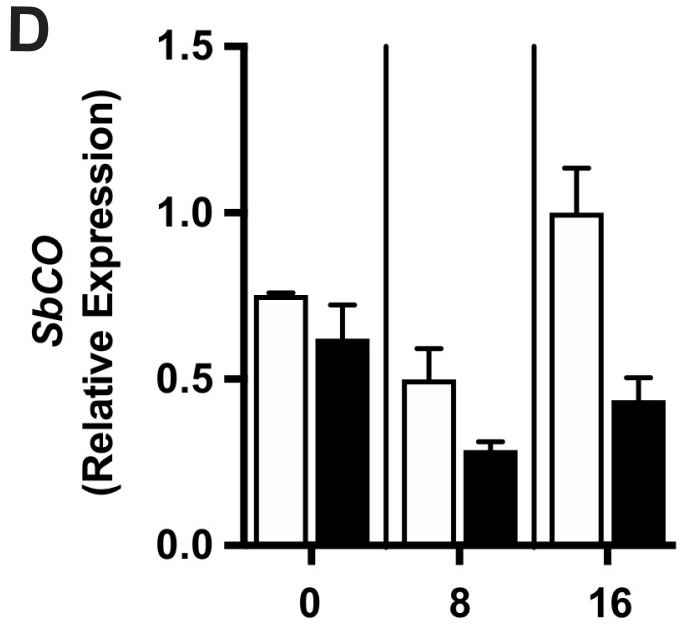

Time after dawn, hours

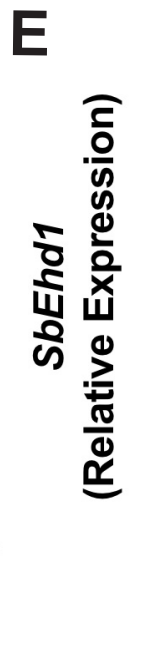

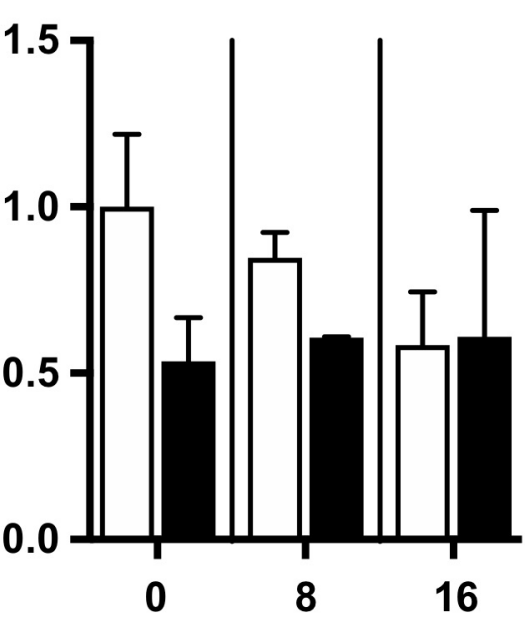

Time after dawn, hours

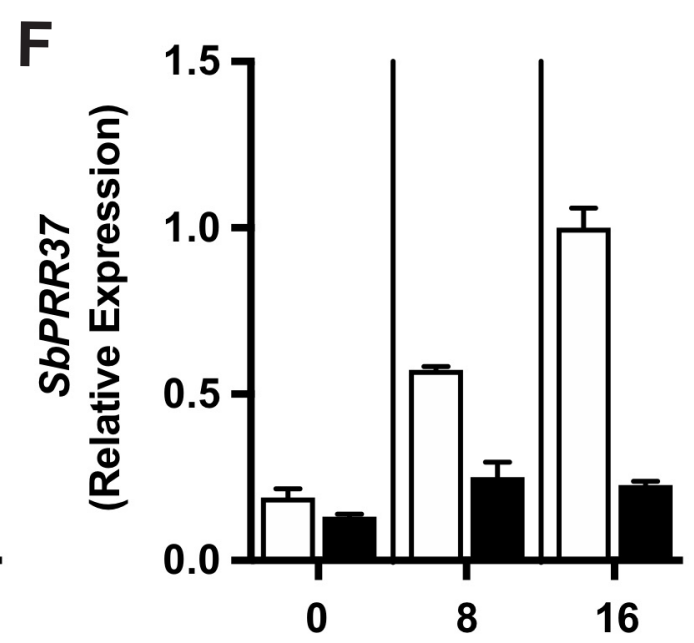

Time after dawn, hours

Figure 4. Sbgi-ems1 alters flowering time gene expression patterns and levels. Transcript levels for SbCN8 (A), SbFT (B), SbCN12 (C), SbCO (D), SbEhd1 (E), and $S b P R R 37(\mathrm{~F})$ in leaves of normal (white bars) and Sbgi-ems1 (black bars) BC1F3 plants at $6^{\text {th }}$ leaf stage grown under LD conditions. Time after dawn is the number of hours after lights on in the morning. Values are the average of two biological replicates normalized to the time point from normal plants with highest transcript levels. Error bars represent the range of two biological replicates. 

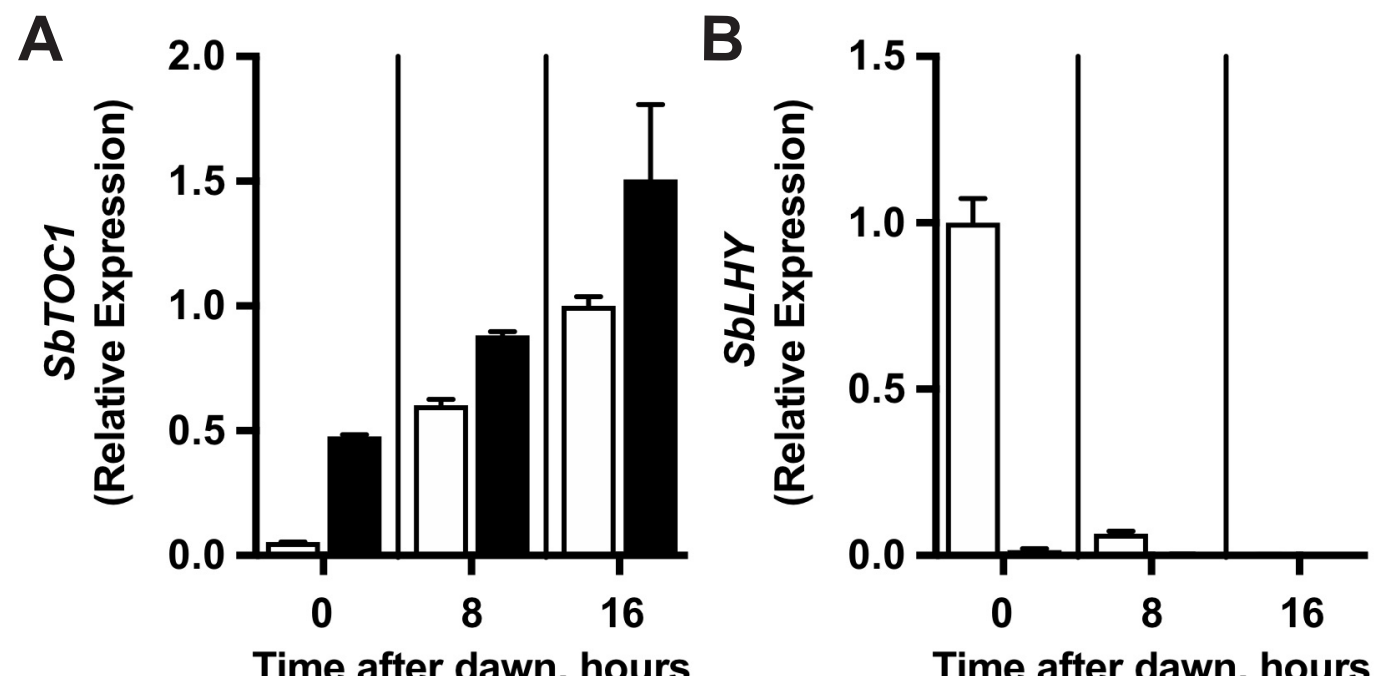

Time after dawn, hours

Figure 5. Sbgi-ems1 disrupts SbLHY and SbTOC1 expression. Transcript levels for $\operatorname{SbLHY}(\mathrm{A})$ and SbTOC1 (B) in leaves of normal (white bars) and Sbgi-ems1 (black bars) BC1F3 plants at $6^{\text {th }}$ leaf stage grown under LD conditions. Time after dawn is the number of hours after supplemental lights came on in the morning. Values are the average of two biological replicates normalized to the time point from normal plants with highest transcript levels. Error bars represent the range of two biological replicates. 